\title{
VR or not VR - an eeZee question?
}

\author{
Wolfgang Dokonal \\ Graz University of Technology, Austria \\ Dokonal@tugraz.at \\ Mike Knight \\ University of Liverpool, UK \\ M.W.Knight@liverpool.ac.uk
}

\author{
Ernst Dengg \\ Graz University of Technology, Austria \\ Dengg@tugraz.at
}

\begin{abstract}
Virtual Reality (VR) software has developed to the point where, for the architect who is averagely technically adept, it can be incorporated into the design process with reasonable effort and costs. For VR to be an effective design tool, it must add value to the design process and should give insights and opportunities not available by other methods. Previous research by the authors reported on the results of an international student workshop, which focused on both the workflow, and the spatial perception that users experienced. In this paper, we continue to explore the question: "Can low cost VR be an effective addition to the architects' design toolbox, or does it still remain a "far-fetched, high-tech expensive folly?" We will be assessing both the practicality of integrating VR into the design workflow and the spatial perception of the designer when interacting with the model. We are experimenting with additional interface tools for the new low cost Head Mounted Displays.
\end{abstract}

Keywords: Virtual reality; Google Cardboard; Low cost interface; Oculus Rift

\section{Introduction}

2016 has the potential to become a turning point for architects in the use of VR and/or Augmented Reality (AR) in the design process. VR has been on the agenda for a very long time but has never really managed to have a significant impact on the design workflow of an average sized architectural practice. The reasons are quite clear: up to now, a substantial amount of investment, both financial for the equipment and time was necessary. Complex workflows to bring the geometry into the systems together with complex technical settings with many technical issues (e.g. synchronization of different PCs, projectors etc.) did not help to establish VR. A lot of preparation was necessary to get anything out of it. After all this work, the main benefits were still unclear - at least outside of academia and the Global companies. There has been a considerable amount of research done which is concerned with high cost and large VR installations such as Powerwalls and CAVEs but for the 'average' rather small architectural practice in Europe, these have been of little practical use.

Because of that, VR has never been a viable option for most architectural firms. Most of them would not have considered using VR even for final project presentations and even less for using it during the design process - mainly because of the reasons mentioned above. This statement is backed up by a survey some of the authors did in the past about the use of digital tools in the design process in architectural firms in Austria and England. (Dokonal and Knight, 2007)

Recent advances and developments in low cost VR hardware (e.g. Head mounted displays HMD) and software, in particular those that use mobile phones as a computational head mounted device, have given all architects and designers the opportunity to use VR as an effective part of the design process.

The new changes in the whole system are driven by the interests of the gaming industry and today, this a powerful and economically flourishing industry with a great deal of available resources.

With the new devices that are already available, or will be available very soon, the "game" is changing and we think that architects should finally make use of this opportunity. With the Oculus Rift Consumer Version, Sony's Morpheus, HTC's Vive, Samsung's Gear and the improved Google Cardboard among others, there is a wide range of extremely low to very low cost VR devices available for the general public. Microsoft HoloLens is now available for developers in the US and Canada and seems to have a lot of potential. In our opinion, it is now the right time for architects to rekindle an interest in these systems because they have the potential to add a new level to the design as well as the presentation process. Together with free or low cost software and an easy to use workflow, there is the opportunity for everyone who is interested.

As in many areas, the car industry leads the way. Car manufacturers have been using expensive and complex VR systems as an integral part in the design process for decades and are now starting to use the low cost VR versions for giving potential buyers an immersive experience in configuring their future car. However, architecture is starting to catch up: The Prefabrication - Housing industry is starting to show an interest in these new devices and there are some examples of how they make use of the low cost VR and AR systems. The APP Roomle from an Austrian producer is a good example. (https://www.variohaus.at). In addition, the new Zaha Hadid project for Graz already uses VR and AR 
technologies for smartphones - see the "Argos Graz" App to visit the unbuilt project

\section{Relevant previous work}

This current research continues work started in 1999 on low cost architecturally appropriate interfaces for VR. (Knight and Brown, 1999). This took the form of a modified exercise bike to 'cycle' around VR models. Whilst the interface worked in increasing the degree of immersion that the user felt, Head Mounted Display (HMD) technology of the time only existed in a very high-end form so the image used was a large format projection. The availability of cheaper HMDs makes revisiting and updating this research appropriate. The experience gained here into what is required to improve the sense of immersion was of use in this current research. One key finding was that naturalistic interface methods ameliorated some of the motion sickness that users experienced.

\section{The 2016 systems}

The current research focuses on the use of smartphones with Google Cardboard and the Oculus Rift systems. We used two approaches to bring the 3D geometry models into the VR systems.

The first was to use the game engine software 'Unity3D' to prepare the 3D geometry for the Oculus Rift systems. We also used Unity3D to create Google Cardboard versions for Android phones. Unfortunately, we could not create iOS versions due to Apple requiring (paid) developer registration. The second approach was to use the app 'Kubity' (www.kubity.com). This provides a cloud-based service where 3D models are uploaded to a server and prepared automatically for the use in combination of Android smartphones in 2D panorama form and Google Cardboard devices for a full 3D interactive environment. This is a straightforward process where the geometry is uploaded and a quick response code ( $Q R$ code) is displayed on the PC screen as a link to the uploaded model in VR. The QR code is scanned from the smartphone app and the model is downloaded to the phone. This sounds perfect and in some ways, it is, but it comes with a number of disadvantages. There is no collision detection implemented yet and there are some problems with the movement inside the models - we will discuss this problem in more detail later.

However, development in this field is very fast and we can assume that there will be updates to 'Kubity' that will resolve some of the known issues - e.g. a recent update adds the possibility to use the open wavefront obj file format. Currently the service is free, but it might be expected to be monetized at some point.

There are probably also some issues of data security to be considered when architects use this method in the design process that are more important. We assume that there will be a certain reluctance to upload preliminary designs to an unknown server when working as an architect on a project or competition. For this purpose, there were no issues for the models we used.

\section{The task for the students}

As last year, we wanted the students to model their own flat using either SketchUp or other modelling software; the assumption being they would have a good knowledge of their own living environment. Because of that they are very good candidates for experiencing these "real world" environments in the VR systems and give feedback about their experience using them.

To give an extra 'virtual' dimension to the research process we added two small built projects that we had received from an architectural practice so the students could also experience projects unknown to them. These projects were two houses in rural locations in the UK. To keep the scale similar to their own flats, only part of the houses were modelled. Unfortunately, the designers themselves could not be part of the workshop but we gave them the VR environments in smartphone form for them to experiment with and provide feedback on the spatial experience that it gave them.

\section{The workshop this year}

The workshop was structured in a similar manner to last year and was again taken by Masters and undergraduate students from TU Graz (Austria) and the University of Liverpool (UK). During the first phase in Graz, students modelled their own flats using SketchUp. During the second phase in Liverpool, students developed their models further in Unity3D and then tested them using VR equipment. They also completed a questionnaire which recorded their experience and accuracy of spatial perception. One difference this year was that the VR software/Google Cardboard integration had developed rapidly over the year since the previous workshop. This resolved some, but not all of the problems encountered.

\section{Problems, Possible solutions and the questionnaire}

We experienced some of the same problems as last year (see "New Interfaces - Old Models" Dokonal, Knight, Dengg, 2015) together with some new ones.

One of the main problems we encountered last year was the import and translation of the SketchUp models into Unity3D. We were looking at the use of Okinos Nugraf as a means of translating/preparing the files, but this has been superseded by the inclusion of a direct SketchUp importer in Unity itself. This meant we had far fewer problems in bringing the geometry into the systems, both with Unity and 'Kubity', which also directly translated for Cardboard. The need for a well-disciplined and constructed 3D model remains, which some of the students found to be a greater challenge than VR itself. After some time and some reworking, all students were able to experience their models virtually.

The problems this year were not in the model import, but in navigating around the models in VR. There are different ways to navigate in the Virtual World using the HMD's. As in the Cardboard demos, the user can look in a given direction and then focus a certain amount of time on a point to move 
towards that point. Although this kind of movement is sometimes quite convenient, there is no equivalent yet to this kind of movement in the real world. So it does not really add to the feeling/perception of immersion.

With Unity and Kubity, the user can trigger a switch to take a step in the virtual world. The first generation of the Google Cardboard device had a magnet attached that activated the switch. This was very unreliable and worked in some cases with some smartphones but not always. The second Cardboard version replaced the magnet with a mechanical button that produced a tap on the smartphone screen.

To move forward inside "Kubity" it is necessary to use the second version - a tab on the screen of the smartphone rather complicated with the first versions of Google Cardboard we had available for the workshop.... To use the apps produced by Unity3D we had to use the unreliable magnetic switch to move inside the VR model. The original plan was that everyone should use her or his own phone but that turned out to be a major problem. Although most of the phones should have had the same set of sensors, they all behaved very differently.

It is worth restating that one of the main goals of the exercise was that students should be able to use the workflow we developed during these workshops to use the VR app as a design tool for their own projects in the future. Being dependent on specific models of smartphones is a major drawback. Having many problems of navigating inside a VR model is not very helpful in terms of immersion and leaves the user with an experience of frustration rather than immersion.

We had a great deal of discussion on how to overcome these kinds of problems. One of the possible solutions we called the 'mechanical finger' because we discussed that the simplest way would be to have a mechanical finger which does the tapping on the screen when the VR device is in use - ideally connected to the feet. It was not possible during the short workshop to get all the necessary parts for our ideas to build it. The workaround for the limited time available during the workshop was to use only the smartphones that were working more reliably with the magnetic switch.

The result of the questionnaire is not significantly different to previous results and might have been influenced by the fact that we severely struggled with navigation problems.

So that we could directly compare results with last year's workshop, the same questions were asked again; the results (2015 figures in brackets) were almost identical - (see Dokonal and Knight 2015).

- Viewing in a VR system helped to understand the design better 95\% (94\%)

- Spatial perception was much clearer and closer to reality than a 3D CAD model $96 \%$ (94\%)
- $\quad$ They could easily orientate themselves in the flats $92 \%(90 \%)$

Where the survey differed was in two areas. Firstly, the question about the 'atmosphere' created was much more positive. This is due to being able to create much more realistically rendered models than the 'white card' materials that we used last year and the need for more detail/materials was again reduced as a requested improvement. However, the lack of realism in the movement methods in Kubity (i.e. lack of collision detection and gravity) meant that some respondents felt that this affected the degree of immersion in the experience.

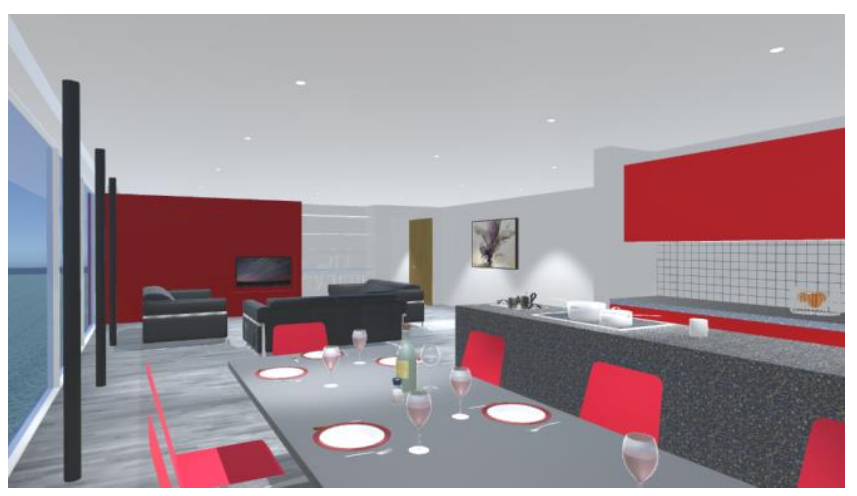

Figure 1: Modelled interior of unfamiliar house

The discussions during the workshop inspired the thought that the Cardboard VR device would gain a lot in terms of immersion (besides all those other aspects like level of detail etc.) if a truly naturalistic navigation method could be devised - i.e. moving freely inside the virtual world just by walking.

As we wanted to have it as a low cost / DIY version within our 'workflow for everyone', all the sophisticated systems using cameras and other tracking devices were not an option. We wanted a solution available on a student's budget and within their capability to construct. Moreover, we did not want to limit our walking range like the Oculus Rift (Tracking area $1.75 \mathrm{~m} \mathrm{x}$ $4 \mathrm{~m})$ and the HTC Vive $(5 \mathrm{~m} \times 5 \mathrm{~m})$ and the physical cage of CAVES, respectively. This is one of the biggest advantages of our system.

Thankfully, Google also seemed to have realized that the magnetic switch was not reliable enough and the second version of the Cardboard viewer replaced the magnetic switch with a simple kind of mechanical finger extension to make it possible to tap on the screen with the device on.

Therefore, our mechanical finger only had to synchronize feet movement to a tap on Cardboard and not on the smartphone itself, which reduced the risk of the infamous Spiderweb App (broken screen) significantly. The solution was to equip Cardboard with a solenoid and connect it to switches on both feet. To keep it simple we started with a wired version. 


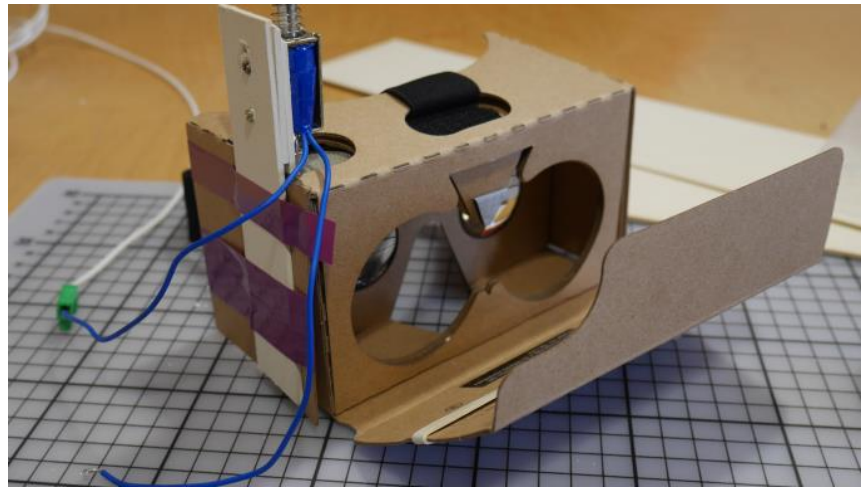

Figure 2: Cardboard with Solenoid

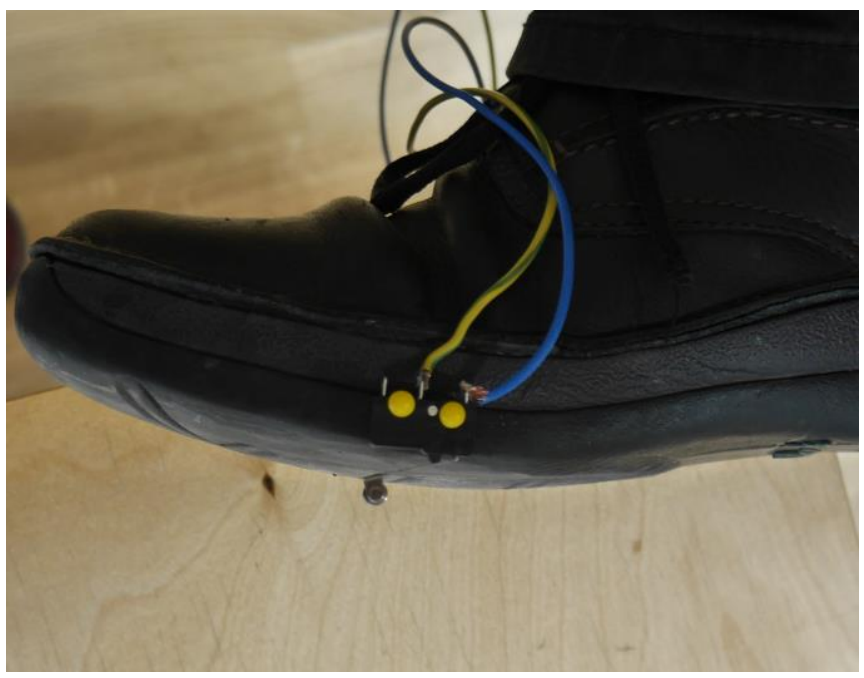

Figure 3: Micro switch mounted to shoes

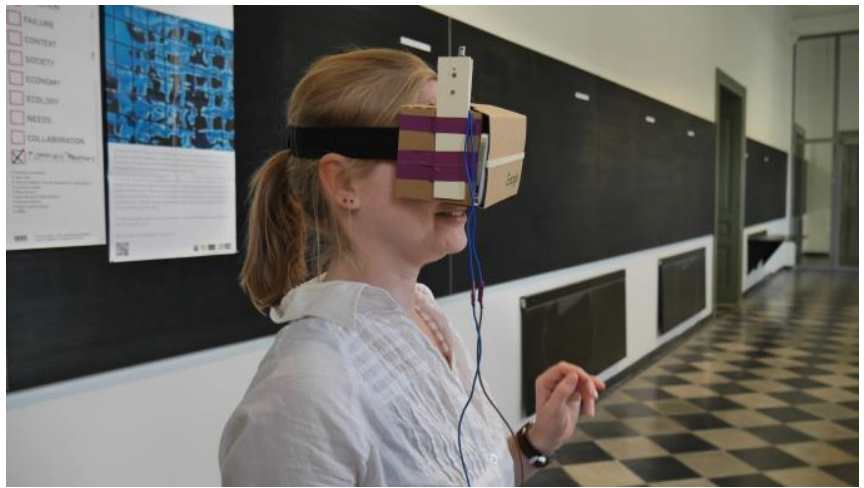

Figure 4: Testing of "eeZee click"

Now the solenoid is mounted to the Google Cardboard with Cardboard and Gaffa tape and the switches are pinned to the soles of both shoes of the person using the device. A 12-volt battery pack produces the energy for the solenoid. The circuit is wired in such a way that the solenoid is pushing the Cardboard tab whenever the person puts both feet on the ground. That was the easiest way to manage the movement without any additional electronics - there are other options in rewiring that we will discuss later. One of the problems with that circuit is that if the user stands with both feet on the ground the tab is constantly activated. This is no problem for the Virtual world - you simply stop and stand as in the real world. However, it is a slight problem in the real world because the solenoid is working constantly and therefore overheating.

\section{First tests with the "eeZee click"}

The result of our mechanical finger is quite stunning - it simply works. It makes a big difference to be able to move in the virtual world by just walking in the real world. Nevertheless, it is vital that the user makes sure that the real world is big enough to move around without banging heads.

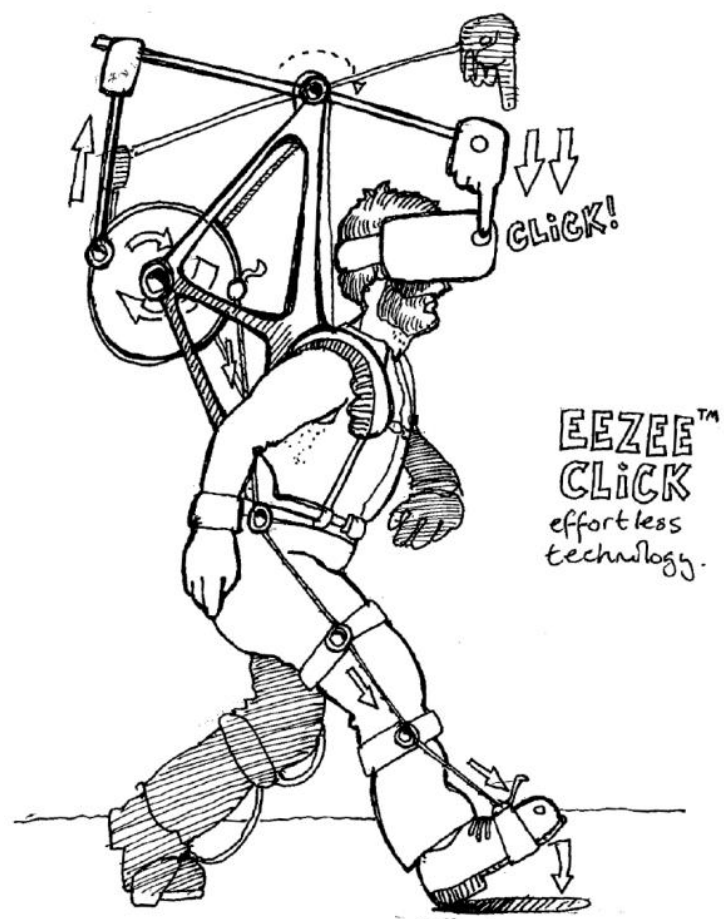

Figure 5: eeZee click concept (with apologies to Heath Robinson)

Still there are some problems in movement using the Kubity app. First, there is the problem of a lack of real gravity or physics. This means that you cannot fix the eye level to a constant height that equates to eye level. So if you look up or down during walking you end up either flying up in the direction that you are looking or digging in the ground. Whereas flying is sometimes quite useful, it is not so nice to end up underneath your model. Another issue is that there is no calibration for the width of any step inside the VR model when you use Kubity now. It worked quite well with all the models we tested but now we would have to rescale the model and re-upload it to Kubity if it is necessary to make any changes. This should be no problem when we use Unity3D to produce Cardboard apps out of the geometry but this has not yet been fully tested. 


\section{Outlook for "eeZee click"}

We discussed several ideas what new possibilities our enhanced device could have with different settings - some of them are not really within architecture.

At the moment with our serial circuit, we can only walk not run. Whilst this is no problem to experience a house or a flat in VR for normal purposes (normally you do not run in a flat) it might be interesting for special tasks inside houses. For example, to test the design of an escape route in the Virtual world, it should be possible to run as nobody just walks away from a fire. Being software for the gaming industry, Unity3D offers many opportunities for fire and explosions that can be scripted as events into the geometry. Therefore, this could be an opportunity to test the ability of different groups to escape certain geometry in a specified time (but always ensuring that there is enough space in the real world...)

Another situation for the use of running in the Virtual Model would be the experience of a city model like a jogger, which also gives a different experience.

For all these cases, we would need to be able to switch the circuit to parallel mode, which should also be possible within our low cost approach. This switching of navigation modes is possible by creating on onscreen interface that would allow the choice of walk/run/Superman modes. This is planned for the next version.

\section{A different interface approach - Xbox controller}

The second interface method used a standard Microsoft Xbox controller. Whilst this removes the common problem of the user being able to move the head though 360 degrees whilst using a static keyboard, it required modification to allow a natural walking movement.

The controller is supported by the Unity Interface controller and by Android smartphones, so it was a simple matter to map two buttons to a script, which moved the camera onestep per activation. Two push buttons were installed on the heels of the users' shoes, and the wires extended to the Xbox controller. The Xbox controller was attached to the Android smartphone with a USB OTG cable. If the user wants to move by 'walking on the spot', this 'walking' movement is achieved by alternatively raising and lowering each heel in turn. Turning is a matter of swiveling on the balls of the feet. It is also possible to walk naturally, but a 'second' is required to prevent accidents such as walking into physical objects. The Xbox controller method allows for more flexibility and adaptability than 'eeZee click' ${ }^{\top \mathrm{m}}$, but is more complex to initially setup. The advantage of using Unity is that it has a full physics engine that allows a more natural movement and interaction with the virtual objects, but again, these require setting up a more complex workflow.

\section{Back to the old days - a project for the future - the exercise bike with HMD devices}

In working on this project, it was also decided to revisit early VR work by some of the authors, particularly the exercise bike. (Knight and Brown, 1999/2000/2001) Some of the problems encountered on this can now be resolved because of advances in both the flexibility and capability and the costs of professional quality game authoring software, which can be used for this purpose.

One of the main problems with the original bike was the handlebar movement, which was achieved by adapting an old Microsoft mouse using gear wheels designed for radiocontrolled models. The problems arose not in the physical side, but in a lack of flexibility in configuring the software. Small movements were fine, but anything more than 45 degrees meant rotating the handlebars a full 360 degrees not a natural movement. At that time a large-scale projection screen was used in lieu of a headset, the most natural navigation method was to use the bars. However, this gave other problems as the projection screen was in a fixed position. With the flexibility of Unity3D, it is now possible to correct these problems and, when coupled with the use of a HMD, produce a much more natural and immersive experience.

In revisiting the bike to integrate it into the new interfaces like "eeZee click" and using head mounted devices, we expect to overcome these problems. By using a head mounted display, the navigation system can be greatly simplified. Looking in the direction of travel will replace the movement of the bars and for the forward movement, replacing the optical sensor with physical switches raises the possibility of a more complete system. There a user can 'walk' around a smallscale environment and then use the bike to 'cycle' at a faster speed around a larger scale (e.g. urban environment). To use the exercise bike as an interface would be especially good for experiencing city models by cycling through them in the Virtual Environment - as the user does not have to worry about crashing into a wall in the real world.

The original nAVRgate intention was to increase the sense of presence in a VR environment through the interface. Our new interfaces in combination with the HMD are doing exactly this. We are now discussing several options about a seamless transition from walking with "eeZee click" to using the exercise bike so that we can simulate a virtual trip from inside a flat into the city and vice versa in a most natural way.

\section{Conclusions}

The new low cost HMD has great potential in architecture and will be part of the designer's toolbox in the future - at least in our opinion. We are now at a moment in time when the game changes. Compared with the development of the internet, which had an extra boost with the development of smartphones, we are getting to a point where VR also will become a boost as a design assistant. 
The switch from CAVE's and Powerwalls to Head Mounted solutions makes an incredible difference - not only financially but also in terms of usability for the less IT literate architect. Now you can "play around" without having to consider a serious investment and weeks of training.

The main questions that we tried to answer are:

- Do (low cost) VR systems improve the perception of space for designers in the early design phases?

- Is there a straightforward workflow that architects without programming experience can use to bring their designs into the new head mounted VR systems easily?

- How immersive are these new low cost systems compared to expensive VR systems (e.g. caves and Powerwalls)?

- What impact do different types of movement control have on the quality of the immersive experience?

We have no clear answer to all of these questions yet but we have clear expectation what these answers will be.

There is no doubt that the simplicity and almost low-tech approach of 'eeZee click' or the Xbox controller together with the fact that it works without being dependent on the sensors in the smartphones is one of its biggest advantages. With these interfaces the new HMD's can be used with any average smartphone and therefore enabling anyone to view their designs in a VR environment with a minimum of both time and financial investment.

There are currently limitations to Kubity (now qrVR), but this is to be expected in a new application. Of more concern is the data security issue of sending (and possible storage) of potentially sensitive design data on a remote server. Therefore, in the end, the perspective to use 'eeZee click' or the Xbox controller with Unity creating a native Cardboard app is the workflow that we really want to establish. We are still experiencing some problems with this workflow that we have not completely solved. One of the big problems is the update cycles of the different software - in this mainly Unity, and Cardboard. The whole area is developing rapidly so you do not really know what you can expect when you use Applications for Smartphones that are freely available. The problem is that you never know what you get when an Application you use for a certain task is doing an update.

During the project with the students, we realized that all our Kubity installations had been updated to a new App called "qrVR". The new App had new ways to navigate inside a VR model. This has certain advantages but it was not working with our "eeZee click" device. Although the movement in the new app was easier and quicker it did not give the same amount of immersive feeling that we create using "eeZee click".

Luckily - After some emails with the developers, a new update of "qrVR" brought back the functionality that we needed for eeZee click.

\section{Outlook}

Although we still experienced several problems during our workshop and the following experimental sessions, the potential of the use of especially the lowest cost smartphone based HMD is obvious to us. Smartphones are ubiquitous today and the costs for the HMD devices very low sometimes they are given away with the phone. Companies already see the potential for clever presentations - see Introduction. Students took to the creation of VR and use of the VR models with comparative ease. They all produced models and were able to see how this can be part of the design workflow. Some tried this by revising and re-visiting their models in the classic circular design>test>revise scenario. This was largely achieved on their own laptops with their smartphones and it was only when the Oculus Rift was used that a higher end computer was required. Most of the participants indicated that they would at least try this on their next design project and a smaller number were very enthusiastic.

After adding the eeZee click interface to Cardboard, the students were even more enthusiastic about working with these devices. Bringing together free or low cost software, some carefully selected cheap components, some ingenuity and an easy to follow workflow will enable us to establish VR in the design process. In our opinion, VR in the design process will be no longer a question, it will be a reality soon for students and for the "average" architectural firm, VR will be a valid tool in the designer's toolbox. We think that our new low cost interfaces for the low cost head mounted displays have the biggest potential to increase the use of VR outside academia and the "Global Players".

With thanks to Darren Ward at RedRaven Design (www.redraven.net) for permission to use the Long House in Cockermouth for testing and Ben Devereau for the eeZee click concept sketch.

\section{References}

Miltiadis, Constantinos (2015) Virtual Architecture in a Real-time, Interactive, Augmented Reality Environment - project Anywhere and the potential of Architecture in the age of the Virtual, Martens, B, Wurzer, G, Grasl T, Lorenz, WE and Schaffranek, R (eds.), Real Time - Proceedings of the 33rd eCAADe Conference - Volume 1, Vienna University of Technology, Vienna, Austria, $\begin{array}{llll}16-18 & \text { September 2015, p1-68 }\end{array}$ http://papers.cumincad.org/cgibin/works/Show?ecaade2015_113

Af Klercker, Jonas and Henrichsen, Jan (2001) Can simulations in VE support architects in solving complex design problems? , Stellingwerff, Martijn and Verbeke, Johan (Eds.), ACCOLADE Architecture, Collaboration, Design. Delft University Press (DUP Science) / ISBN 90-407-2216-1 / The Netherlands, pp. 77-82

Anette Kreutzberg (2014) New Virtual Reality for Architectural Investigations, Thompson, Emine Mine (ed.), Fusion Proceedings of the 32nd eCAADe Conference - Volume 2, Department of Architecture and Built Environment, Faculty of 
SIGraDi 2016, XX Congress of the Iberoamerican Society of Digital Graphics 9-11, November, 2016 - Buenos Aires, Argentina

Engineering and Environment, Newcastle upon Tyne, England, UK, 10-12 September 2014

Dokonal, Wolfgang; Knight, Michael W. and Dengg, Ernst Alexander (2015) New Interfaces - Old Models. Martens, B, Wurzer, G, Grasl T, Lorenz, WE and Schaffranek, R (eds.), Real Time Proceedings of the 33rd eCAADe Conference - Volume 1, Vienna University of Technology, Vienna, Austria, 16-18 September 2015, pp. 101-106

Krakhofer, Stefan and Martin Kaftan (2015) Augmented Reality Design Decision Support Engine for the Early Building Design Stage, Emerging Experience in Past, Present and Future of Digital Architecture, Proceedings of the 20th International Conference of the Association for Computer-Aided Architectural Design Research in Asia (CAADRIA 2015) / Daegu 20-22 May 2015, pp. 231-240

Kreutzberg, Anette (2015) Conveying Architectural Form and Space with Virtual Reality, Martens, B, Wurzer, G, Grasl T, Lorenz, WE and Schaffranek, R (eds.), Real Time - Proceedings of the 33rd eCAADe Conference - Volume 1, Vienna University of Technology, Vienna, Austria, 16-18 September 2015, pp. 117124

Shemesh, Avishag; Moshe Bar and Yasha Jacob Grobman (2015) Space and Human Perception, Emerging Experience in Past, Present and Future of Digital Architecture, Proceedings of the 20th International Conference of the Association for ComputerAided Architectural Design Research in Asia (CAADRIA 2015) / Daegu 20-22 May 2015, pp. 541-550

Abdelhameed, Wael A. (2014) Creativity and VR Use, Rethinking Comprehensive Design: Speculative Counterculture, Proceedings of the 19th International Conference on ComputerAided Architectural Design Research in Asia (CAADRIA 2014) / Kyoto 14-16 May 2014, pp. 719-728

Freitas, Márcia Regina de and Regina Coeli Ruschel (2013) What is Happening to Virtual and Augmented Reality Applied to Architecture?, Open Systems: Proceedings of the 18th International Conference on Computer-Aided Architectural Design Research in Asia (CAADRIA 2013) / Singapore 15-18 May 2013, pp. 407-416

Knight, Michael and Brown, Andre (1999) Working in Virtual Environments through appropriate Physical Interfaces; Architectural Computing from Turing to 2000 [eCAADe Conference Proceedings / ISBN 0-9523687-5-7] Liverpool (UK) 15-17 September 1999, pp. 431-436

Knight, Michael and Brown, André (2001) Towards a natural and appropriate Architectural Virtual Reality: the nAVRgate project. Past, present, future. Proceedings of the Ninth International Conference on Computer Aided Architectural Design Futures [ISBN 0-7923-7023-6] Eindhoven, 8-11 July 2001, pp. 139-149

Moural, Ana; Sara Eloy; Miguel Sales Dias; Tiago Pedro (2013) How Space Experimentation Can Inform Design: Immersive Virtual Reality as a Design Tool, SIGraDi 2013 [Proceedings of the 17th Conference of the Iberoamerican Society of Digital Graphics ISBN: 978-956-7051-86-1] Chile - Valparaíso 20 - 22 November 2013, pp. $182-18$

Knight, Michael and Brown, Andre (2000) Towards Naturalistic Navigation Metaphors for large scale Virt; Construindo (n)o espacio digital (constructing the digital Space) [4th SIGRADI Conference Proceedings / ISBN 85-88027-02-X] Rio de Janeiro (Brazil) 25-28 september 2000, pp. 247-250

Dokonal, Wolfgang and Knight, Michael (2007) Digital Design Tools vs. Sketching in Design, Predicting the Future [25th eCAADe Conference Proceedings / ISBN 978-0-9541183-6-5] Frankfurt am Main (Germany) 26-29 September 2007, pp. 843-848 http://papers.cumincad.org/cgi-

bin/works/Show?ecaade2007_167 